$15^{\text {th }}$ International Conference on

AEROSPACE SCIENCES \& AVIATION TECHNOLOGY,

$\boldsymbol{A S A T}$ - 15 - May 28 - 30, 2013, Email: asat@mtc.edu.eg, Military Technical College, Kobry Elkobbah, Cairo, Egypt, Tel: +(202) 24025292 -24036138, Fax: +(202) 22621908

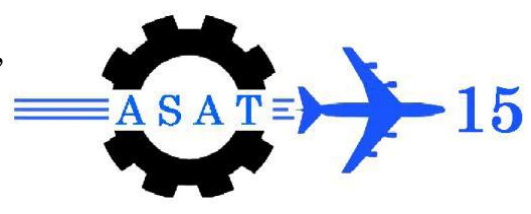

\title{
Thermal Cameras and their Use in Civil Engineering
}

\section{A. Abdelhafiz*}

\begin{abstract}
The modem technique of thermography is originally developed for military use. Infrared cameras use inferred to detect objects temperatures. A Thermal Imaging Camera is used in fire fighting. Such cameras allow fire fighters to see areas of heat through smoke or darkness by rendering infrared radiation as visible light. Thermal cameras have then migrated into civil fields, hi civil engineering, one can get use of this theory to control the energy consumed by Heating and cooling systems in buildings. Therefore new regulations have appeared in some European countries to control the energy consumed by such systems. Buildings insulation efficiency is our concern here, not the heating or cooling systems themselves. In this paper, an integration technique for the thermal image with other sensors is introduced. The integration allows to check the amount of the emitted cool/heat from the concerning building against the accepted limits.
\end{abstract}

Keywords: Thermal cameras, data fusion, laser scanning, digital photogrammetry. insulation efficiency.

\section{Introduction}

Energy generation is a main factor for environmental pollution. Accordingly, energy saving is an important issue for saving the environment. Within the frame work of energy saving in Germany, each building has to fulfil the thermal insulation requirements to get permission for further use through what is called "Energy passport".

Within this passport, certain computer programs are used to calculate the insulation efficiency through the used materials properties. The actual behaviour of such materials can be obvious seen with the help of thermography technique. Buildings openings (doors and windows) can also be checked against ah leakage using the same technique. A thermal image, produced by a thermal camera, is shown hi Figure 1 for a building in Braunschweig. Germany, in whiter (about zero degree temperature).

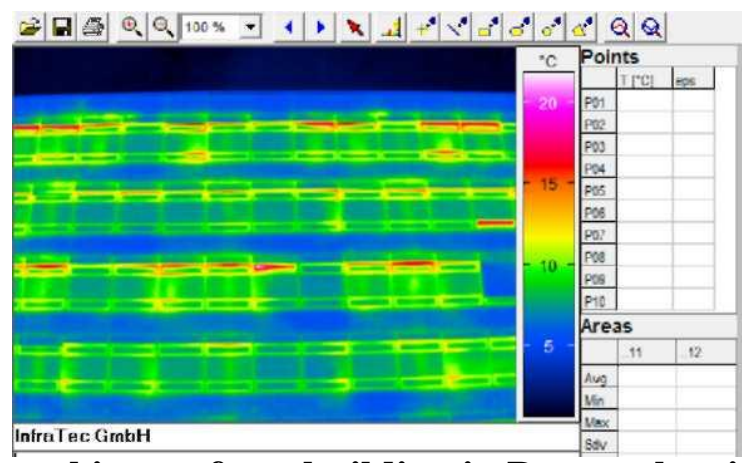

Fig. 1: Thermal image for a building in Braunschweig, Germany

Assistant professor, Civil Engineering Department, Faculty of Engineering, Assiut University, Egypt. a.abdelhafiz@yahoo.com . 
From this image, an overview on the building temperatures is shown. The scale bar on the right side is the temperatures key. The red parts are with high temperatures, i.e. bad insulation. But these windows might be opened, for example, so the heat coming from those windows is normal. An ordinary digital image with the same view can solve this issue. The amount of heat coming from any window couldn't also be computed due to the lake of dimensions. A laser scanner point cloud/mesh will provide the user with all the required dimensions.

In this work, we are concerned with integrating the thermal image, the laser scanner point cloud/mesh and the digital image in order to completely check the efficiency of buildings insulation. A complete check requires the geometry and the visible view of the concerning building to accurately and correctly compute the amount of the emitted heat and consequently check it against the accepted limits.

We aim to deliver a digital archive file for each building containing all the available information (Thermal info / Geometry / prescriptive views). This file helps over years to judge the performance of the building from the energy consumption point of view. Such info can also feed the geographical information system GIS. The main issues here is how to register the three data sets together and then how to integrate them together.

Multiple sensors data fusion are always important to get full use of all the available information about the concerning object. Recently the laser scanner is the common used sensor to recover the object geometry and the digital camera is used to collect the texture. Models with real radiometric characteristics can be then generated by fusing the data sets of these two sensors on the computer screen.

Modern laser scanner types (e.g. Riegl LMS-Z210ii) are equipped with mechanically mounted digital cameras to capture the object texture simultaneously. The attached camera has to be firmly fixed to the scanner body then the relative orientations between the camera and the scanner can be calculated through a certain calibration process. Having relative orientations between both sensors, the corresponding image pixel color for each space point can be obtained. Afterwards point clouds/meshes with the real color can be achieved.

Although the mounted camera approach sounds good, but it still has some limitations [1]. First: the best color quality is obtained at the best lighting conditions of the image which may not be at the same position of the laser scanner. Second: the narrow vertical field of view of the attached camera, around sixty degrees, will limit the wide laser scanner vertical field of view which exceeds the 300 degrees in most modern scanner types.

The pre-mentioned limitations in the camera mounted approach have a great affect on the quality and the flexibility of the obtained results. Therefore the approach of free hand camera positions registered to the point cloud has got a high potential. In this approach, the camera can stand in the best position to give the best color quality, [2]. The photos can also be captured at a different day of the scanning day waiting for better weather for instance. Moreover, photos for pre-scanned objects, which are scanned previously, can be now taken, [3].

The two data sets can be fused using manual techniques [4] and [5] and/or semi-automatic techniques [6] and [7]. It is the method that is chosen for detecting the existing occlusions that distinguishes one technique from another. Interested reader may refer to the followings 
literatures for other combination attempts between the laser scanning technique and the digital photogrammetric technique [8] and [9].

Thermal images are captured by hand held thermal cameras with considerably low resolution (up to 640x512 thermal pixels) comparable with the resolution of the other two sensors used in terrestrial range (the digital camera and the terrestrial laser scanner TLS). Such cameras aren't mounted on scanners till now. The narrow field of view (about $20^{\circ} \times 30^{\circ}$ ) and the high cost might be reasons for that. So, a separate registration step is required to put the thermal images in the coordinate system of the geometry.

In this paper, thermal images are first registered with their corresponding digital images. Afterwards, the digital images are registered with the geometry through interactive selection of points in both the images and the geometry. The three data sets are then presented in a two dimensional environment introducing a new product called Thermal3DImage. The Thermal3DImage interface is the digital photo which can be toggled to the thermal one. Space coordinates for each image pixel with the corresponding temperature are online displayed during the mouse move. Space distances between any two marked points on the digital or the thermal image can be also online measured.

\section{Potential of Thermography}

Thermography technique is based on the scientific principle that all objects of absolute zero (minus $273^{\circ} \mathrm{C}$ ) emit invisible infrared radiation from the surface areas. Warmer objects emit generally greater level of energy. Thermography uses these physical characteristics and translates the infrared signature into a corresponding two dimensional image that depicts accurate surface temperatures.

Infrared thermography can provide remarkable. non-destructive information about buildings like the excessive energy use due to missing or damaged insulation, and excessive air-leakage across the thermal perimeter. Water leakage can also be detected by thermal imagining as the moisture reduces the surface temperature. In roofing, infrared imaging can save building owners too much money by avoiding roof replacement costs. Where thermal insulation becomes fault\}", building construction technicians can see heat leaks to improve the efficiencies of cooling or heating air-conditioning. Thermal imaging photography finds other uses. For example, with thermal imaging, power lines maintenance technicians locate overheating joints and parts, a sign of then failure, to eliminate potential hazards.

Infrared thermography can be divided into two different approaches: passive and active. In the passive approach, materials and structures are sensed at ambient temperature and anomalous temperature profiles or hot spots indicate a potential problem to take care of. In this work, we are interested with sensors use this approach, hi the active approach an external stimulus is used to induce a relevant thermal contrast naturally not present. Depending on the external stimulus applied, different methods have been developed, such as pulse thermography, step heating, lockin thermography, burst-phase thermography or vibrothermography: a review about these technologies can be found in [10].

\section{Technical Specifications of Infrared Cameras}

Thermographic cameras detect radiation in the infrared range of the electromagnetic spectrum (0.9-14 um) and produce images of that radiation. The appearance and operation of a modem thermographic camera is often similar to a camcorder, see different models in figure. 


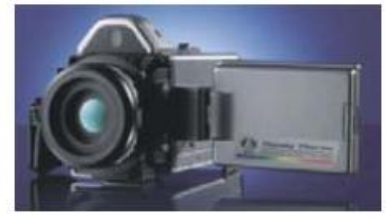

AVIO TVS 200EX

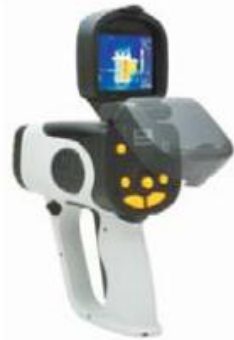

NEC TH7800N

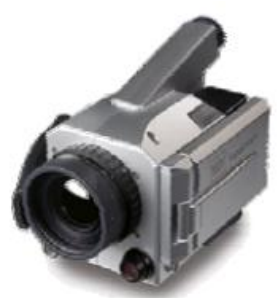

Variocam InfraTec

Fig. 2: Different types of modern thermal cameras

Instead of CCD sensors, thermal imaging cameras use CMOS (Complementary Metal Oxide Semiconductor) focal plane array (FPA). The newest technologies are using low-cost and uncooled microbolometers FPA sensors. Their resolution is considerably lower than of optical cameras, mostly $160 \times 120$ or $320 \times 240$ pixels, up to $640 \times 512$ for the most expensive models. Thermographic cameras are much more expensive than then visible-spectrum counterparts. More sensitive bolometers require cryogenic cooling, usually by a miniature Stirling cycle refrigerator or liquid nitrogen.

\section{Laser Scanner Point Cloud, Visual, and Thermal Images Registration}

Data registering step aims to define the available data sets (laser seamier point cloud/mesh, visual images and thermal images) in one coordinate system. This system is commonly the coordinate system of the laser seamier point cloud. One may also use a global coordinate system to register the three data sets, hi that case another instrument "total station" has to be employed to measure control points on the concerning object relative to the chosen global system. Afterwards the geometry is registered to the global coordinate system. The images (visual and thermal) are then registered in the same system as well.

In this work, we employ the interactive selection of corresponding points (natural features) technique to register the visual images in the geometry coordinate system. In this technique, the intensity color delivered by the laser seamier is used to visualize the point cloud and consequently to extract the required control points. Natural points (edges or comers) are used as placing artificial targets will limit the flexibility of the approach. Finding well defined natural points in the laser scanner point cloud is not a straight forward task, see figure 3 which illustrates the points extraction process from a point cloud. One can see that there is no laser ray hits the concerning comer and so the possibility to measure the exact comer point doesn't exist. Therefore each operator might assign a different point from the near points.

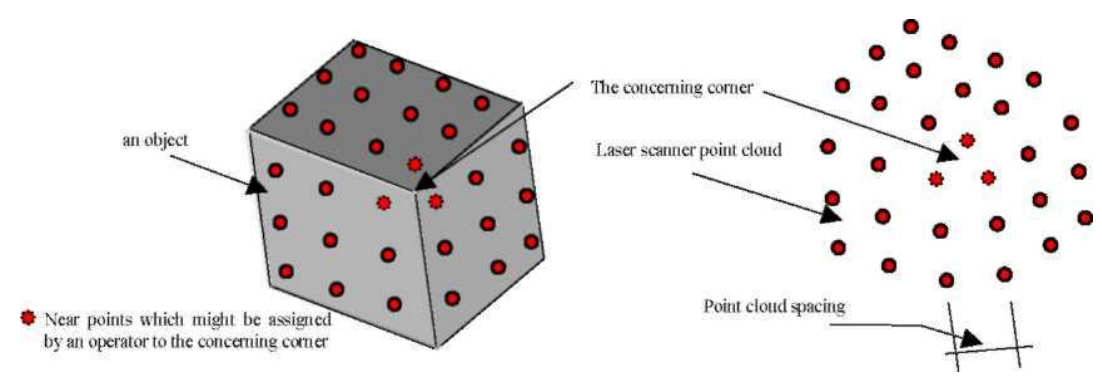

Fig. 3: Extracting space coordinates of a corner from a laser scanner point cloud 
In order to get a photogrammetric solution, Collinearity equations have to be linearized first. In the linearization process, the image coordinates are considered as observations. The interior orientations elements are commonly considered as constants (from calibration). Known and unknown parameters can be then c hanged according to each case. If precise control points are employed, their coordinates are considered as known (error free) and the six exterior orientation elements of the camera are considered as unknown parameters. As a result, precise exterior orientations are expected.

In our case, the extracted points from the laser scanner point cloud are not that accurate. Their accuracy depends mainly on the accuracy of the laser scanner device, the point cloud spacing and the operator interpretation. So, the computed exterior orientations based on those extracted points are expected to be within the accuracy of the extracted points.

Therefore, another registration technique has been used to give the bundle adjustment solution the ability to change the values of the extracted points coordinates during the least squares procedure. This can be achieved by considering the ground coordinates as unknown parameters during the photogrammetric solution. The extracted values are then used as initial values rather than control ones.

Having precise interior orientations from a camera calibration process and close initial values for some ground points extracted from the cloud, close exterior orientations can be then computed. After that the bundle adjustment solution tries to shift and rotate the exposure stations and the bundles of light-rays so that the final results of all unknowns are obtained. By giving close initial values, the bundle adjustment is pushed to converge at the nearest solution which is the right solution. This technique showed reliable results [1], so it will be used to register our point cloud and digital images through this work.

Unfortunately, this technique will not work with the thermal images for the following reasons. Firstly, it is quite difficult to identify corresponding points in the thermal image and their corresponding points in the geometry. Secondly, the measuring accuracy on the thermal images is low due to the low image resolution (maximum 0.3MP till now) and the large pixel size. Thirdly, obtaining the thermal camera interior orientations through ordinary camera calibration process is a very complex task. A calibration trial for a thermal camera can be reviewed in [11]. Even if the calibration is made, thermal cameras are commonly autofocus which will change the camera setting for each photo in both the laboratory and the field. These setting changes prevent computing reliable calibration parameters. On the other hand, the self calibration will not work with such low accuracy measurements (low resolution images).

Therefore the thermal images are registered here with the digital images instead of registering them with the laser scanner point cloud. Having registered digital images with both the thermal images and the point cloud, the thermal images are consequently defined in the point cloud coordinate system as well.

We register here the thermal images with their corresponding digital images through two dimensional transformations. The used transformation is based on six parameters. This type of transformation considers translations, orientations and scale changes in both directions.

At least, three points have to be manually selected in order to perform the desired transformation and consequently achieve the registration. In case of picking more than three corresponding points, the least squares method is employed in order to get the transformation 
parameters. Registering the thermal and the digital images using this technique requires no (thermal) camera calibration. Add to that identifying corresponding points in the thermal images and in the digital images is a more straightforward process. Accordingly the whole task can be carried out simply. In fig. 4, three data sets are registered together in the geometry coordinate system.

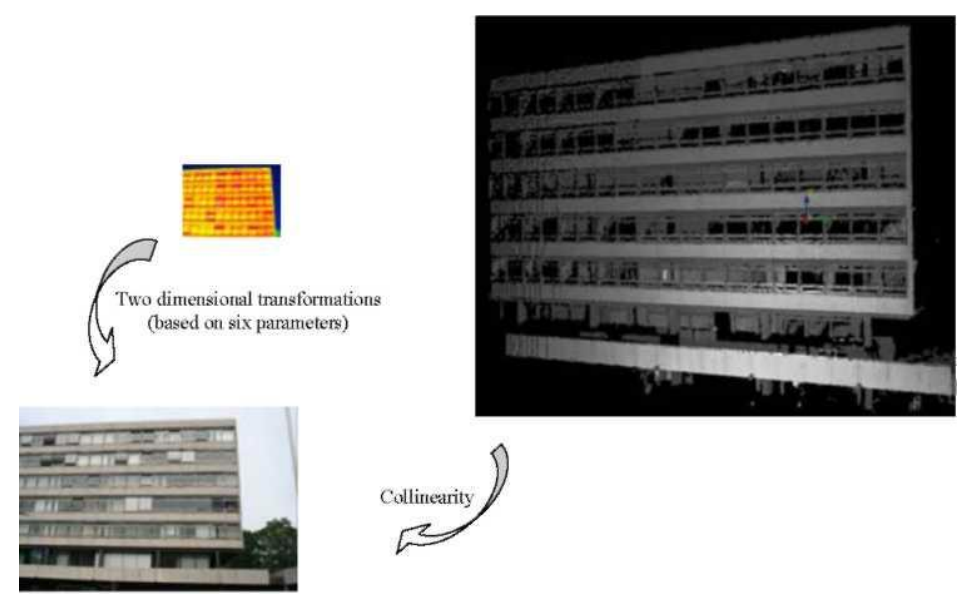

Fig. 4: Three data sets are registered together

\section{Data fusion}

The data fusion aims to define the location of each vertex from the geometry on both the digital and the thermal image. Consequently the corresponding colour and temperature for each vertex are identified. The geometry is projected on the digital image using collinearity equations. The corresponding thermal image pixel for each digital image pixel can be computed through the obtained transformation parameters from the previous registration step. Here, we create a new registered thermal image. The followings are the steps of creating the new registered image:

1) Compute the scale between the visual image and the original thermal image in the height and the width of the image.

2) Select the smaller scale to create the new thermal image (registered) with the same ratio (height/width) of the visual one. All the new image pixels color is white.

3) Project the original thermal image pixels to the new registered thermal image by applying the 2D transformation obtained from the registration step, see Fig. 5.

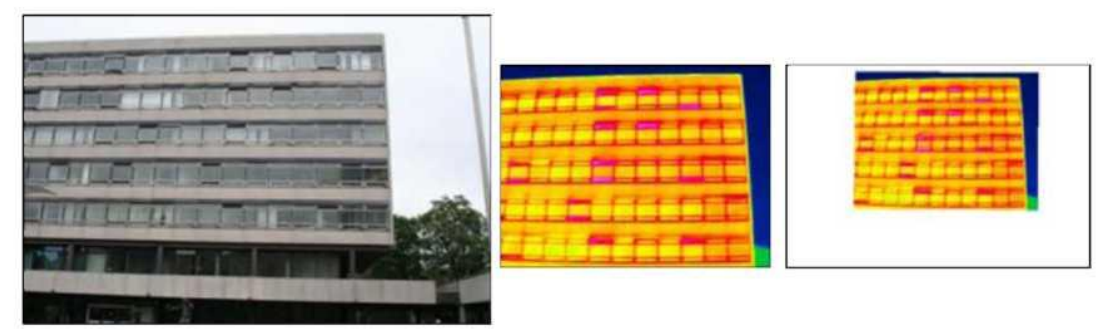

Fig. 5: A visual photo with its corresponding thermal image and the registered thermal image

The new delivered thermal image has the same point of view of the visual image. Each pixel in the new thermal image is corresponding to a pixel in the visual image considering the scale change. All white areas in the new thermal image have no thermal information. 
While occlusions in the scene are considered a serious issue in fusing the geometry with the digital image, no similar problem appears in fusing the thermal image with the digital one as the new thermal image is registered to have the same point of view of the digital image. In case of employing the geometry in form of single point cloud, one digital image has to be captured from the same position of the laser scanner to overcome the occlusions problem.

If multiple point clouds are employed to recover the object, the geometry has to be meshed in order to detect various occlusions in the scene and correctly assign the corresponding image pixel for each vertex. Fig. 6 illustrates the expected occlusions problem in the fusion step when projecting the geometry on the digital image. The basic principles to detect the occlusions automatically are given in the following paragraph.

Where points (A and B) in the figure are occluded, point (C) is visible. According to the fact that the point near to the camera is visible and the far one is invisible, the visibility status for the points can be defined. The two points (A and $\mathrm{C}$ ) are projected on the same image pixel, so the near point to the exposure station $(\mathrm{C})$ is considered visible and the other point $(\mathrm{A})$ is invisible. If a third point is projected on that image pixel, the same procedure will be applied. Finally, one vertex from the model (the nearest to the exposure station) is considered as the correct correspondence for the image pixel.

This will not work with points like B. Such points are stand alone for one image pixel. Those points can be detected as occluded points and consequently removed by considering a mesh model instead of the point cloud. Any vertex will be considered as occluded from a concerning camera point of view if the light ray connecting that point with the exposure station intersects with the object surface.

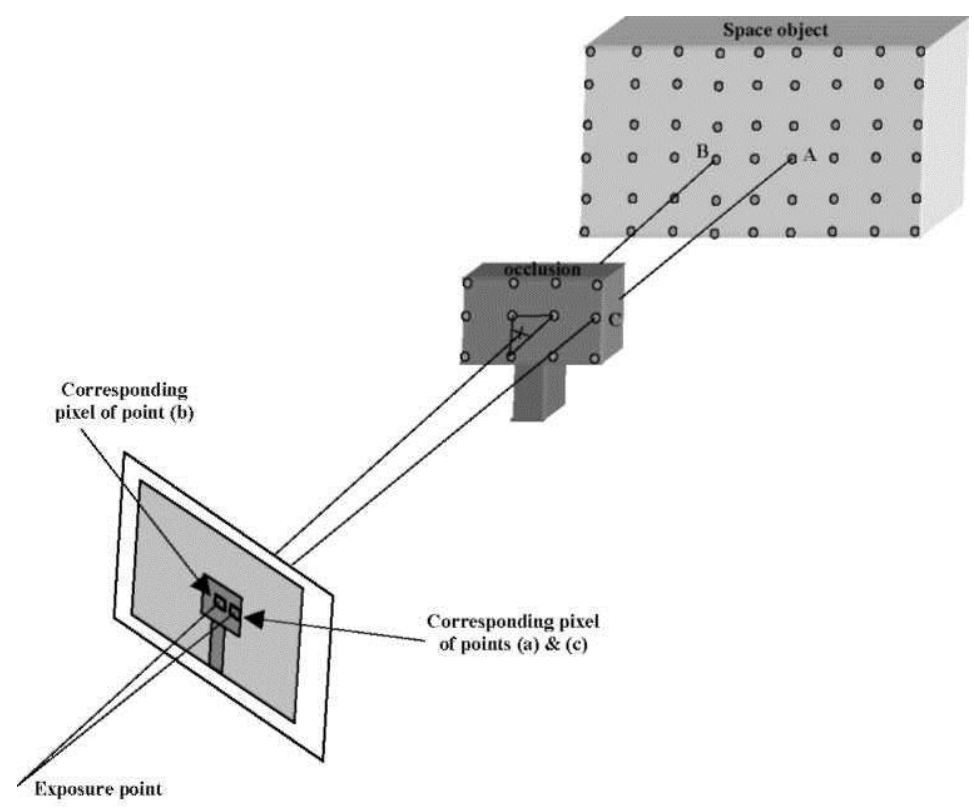

Fig. 6 Occlusions detection

\section{Thermal3DImage}

Digital image textures are commonly warped to the object geometry to produce a three dimensional photo realistic model. Thermal images colors can also be mapped on the geometry to produce a thermal model. The resulted models in both cases bear information from only two data sets. In order to access the third data set simultaneously our Thermal3DImage is developed. 
The Thermal3DImage is a new representation technique, in which the visual image is used as the user interface. The geometry is attached to the visual image pixels to provide 3D coordinates to the image pixels. At each visual image pixel, the corresponding temperature is achieved by knowing the corresponding thermal image pixel (2D transformation), see Fig. 7 top.

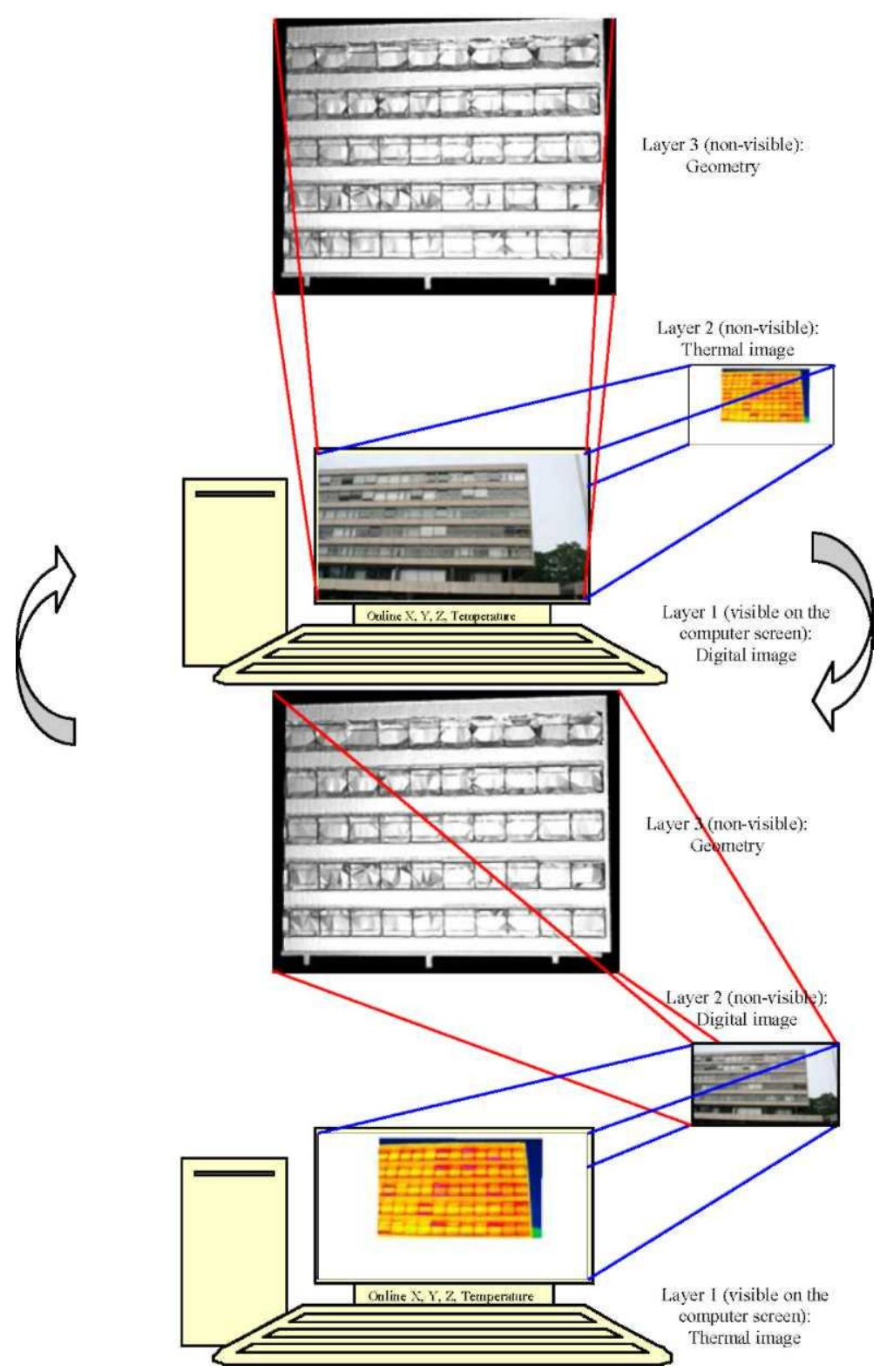

Fig.7: The relationship between the three data sets through the Thermal3D Image, the user can toggle between two modes with one button. In both modes, the space coordinates and the temperature for the targeted pixel are online displayed

The relationship between the temperatures and the thermal colors is given by the thermal camera itself. These colors can also be changed by the user for another type of color presentation. We use here the exported pixels temperatures (numbers) from the IR-camera directly. These temperatures are stored in a matrix. Any value can be then recalled according to the position of the pixel on the thermal image. 
If the thermal image is used as the user interface, see fig. 7 bottom, the corresponding visual image pixel will be computed first. Then the 3D space coordinates are obtained from the attached geometry to the digital image.

Not all the visual image pixels are covered by 3D coordinates, see fig. 8. The 3D space coordinates for the in between image pixels is interpolated online based on the adjacent coordinates. The interpolated surface is influenced by nearby points more than by distant points. Therefore the weight assigned to each scattered point diminishes as the distance from the interpolation point to the scattered point increases. The weight here is standing for the inverse distance. In case of a point cloud is attached, so all adjacent pixels in a given search radius will be considered in the interpolation procedure. In case that a mesh is attached, so only the circum triangle vertexes have to be considered.
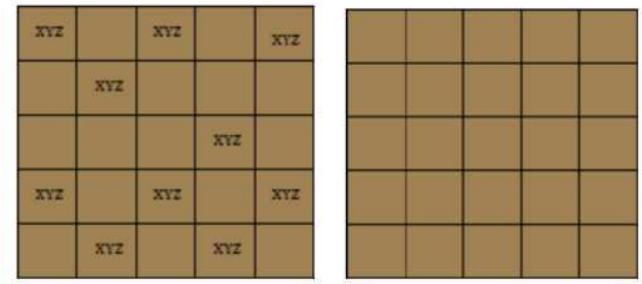

\section{Fig. 8: A laser scanner point cloud and a mesh model are attached to image pixels. One can see pixels without corresponding 3D coordinates which can be calculated based on interpolation technique.}

We employ the 3DImage software, which has been developed by the author in the visual basic programming language, to realize the Thermal3DImage on the computer screen. The data input required to generate a Thermal3DImage for a certain object is:

1) A laser scanner point cloud or a triangulated $3 \mathrm{D}$ mesh.

2) Digital photos from different points of view covering the scene.

3) Thermal images preferably with a view very close to the digital images view.

The user gets the following benefits from the Thermal3DImage:

- While moving the mouse on the interface image, the corresponding 3D coordinates and the temperature of the pixel are displayed.

- The corresponding 3D coordinates and the temperature of a pixel can be written in a file by a mouse click.

- One can toggle between the visual view and the thermal one by a click.

- Two key photos (visual and thermal) appeared on the top-right of the view, by a click at any point on one of them the large view recall that area.

- While moving the mouse on the large view, there are two other cursers moving on the same position on the two key photos.

- Distances between any two marked points can be online measured on both the visual and the thermal image.

- A very useful function that the user can call points with temperatures:

- higher than certain temperature.

- lower than certain temperature.

- lie between two limits.

This function will help detecting any focus of air leakage coming from an opening in the building under investigation. One can also register the visual and the thermal image only 
(without using the geometry) and the previous described function will also work. At that case no 3D measurements will be available on the Thermal3DImage.

\section{Investigating the main building of TU- BS, Germany using the Thermal3DImage}

The developed Thermal3DImage is employed to check the insulation and opening efficiency of the main building of the technical University in Braunschweig, Germany.

\section{Sensors: (see Fig. 9)}

The scan has been performed using the Imager 5003 laser scanner, which uses laser safety class 3R (DIN EN 60825-1) and capable of capturing up to 500,000 points per second. The maximum captured range for this scanner is 53.5 meters. The scanner has a $360^{\circ}$ by $310^{\circ}$ field of view with $0.02^{\circ}$ accuracy. The noise range at 10 meters is 1.3 to 3.3 millimeters, and at 25 meters is 3.0 to 9.0 millimeters depending on the reflectivity from the white to the dark grey. Along with the Cartesian coordinates of each of the scanned points, the laser also measures the intensity value of each point.

The digital images are collected with a professional digital camera Canon EOS 350D, eight mega pixel. The camera sensor dimensions are $22.2^{\mathrm{x}} 14.8 \mathrm{~mm}$ with maximum effective 3456 x 2304 pixels. The camera positions configuration is selected according to the best available photogrammetric configuration rules. The photos were captured with a $24 \mathrm{~mm}$ lens. The lens was adjusted to infinity as in the capturing process and calibrated inside our photogrammetric laboratory in order to get the precise values of the interior orientations.

The thermal images are captured by the VarioCAM® of the German manufacturer JENOPTIK. This type of thermographic cameras is based on state-of-the-art uncooled microbolometer FPA (focal plane array) detectors with 384 x 288 infrared pixels. The VarioCAM ${ }^{\circledR}$ camera is considered one of the efficient tools for buildings insulation check and leakage detection.

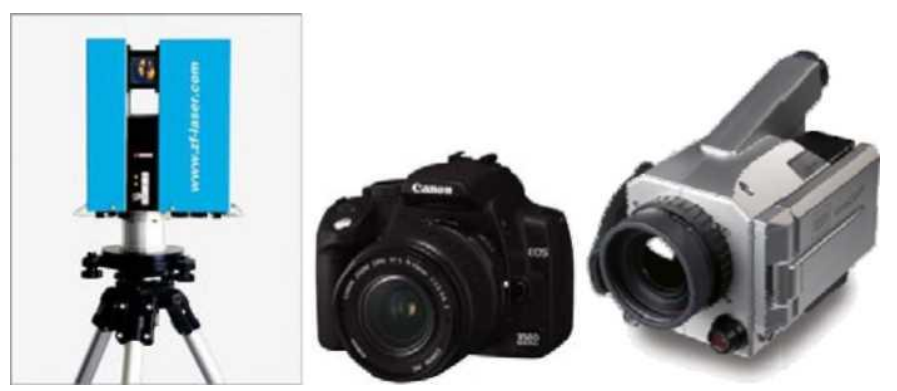

Fig. 9: Used sensors for capturing (The Imager $5003 \mathrm{Z}+\mathrm{F}$ laser scanner - The Canon EOS 350D digital camera - The VarioCAM thermal camera)

\section{Generation of the Thermal3DImage for the Concerning Building:}

The digital images are first registered with the geometry through sixteen natural points. Afterwards the thermal image is registered with its corresponding digital one as shown in Fig. 10.

The three data sets are fused using the Thermal3DImage technique. For each image pixel, its space coordinates together with the temperature are now available online. One can toggle between the thermal and the digital image. Distances on both images can also be measured. 
On the bottom of Fig. 10 , we call points with temperatures higher than 21.5 degrees. One can see that there is air leakage from certain widows. From the visual view, opened windows can be excluded and the user can decide that remaining windows have to be examined; See for example the marked window in the middle of the figure.

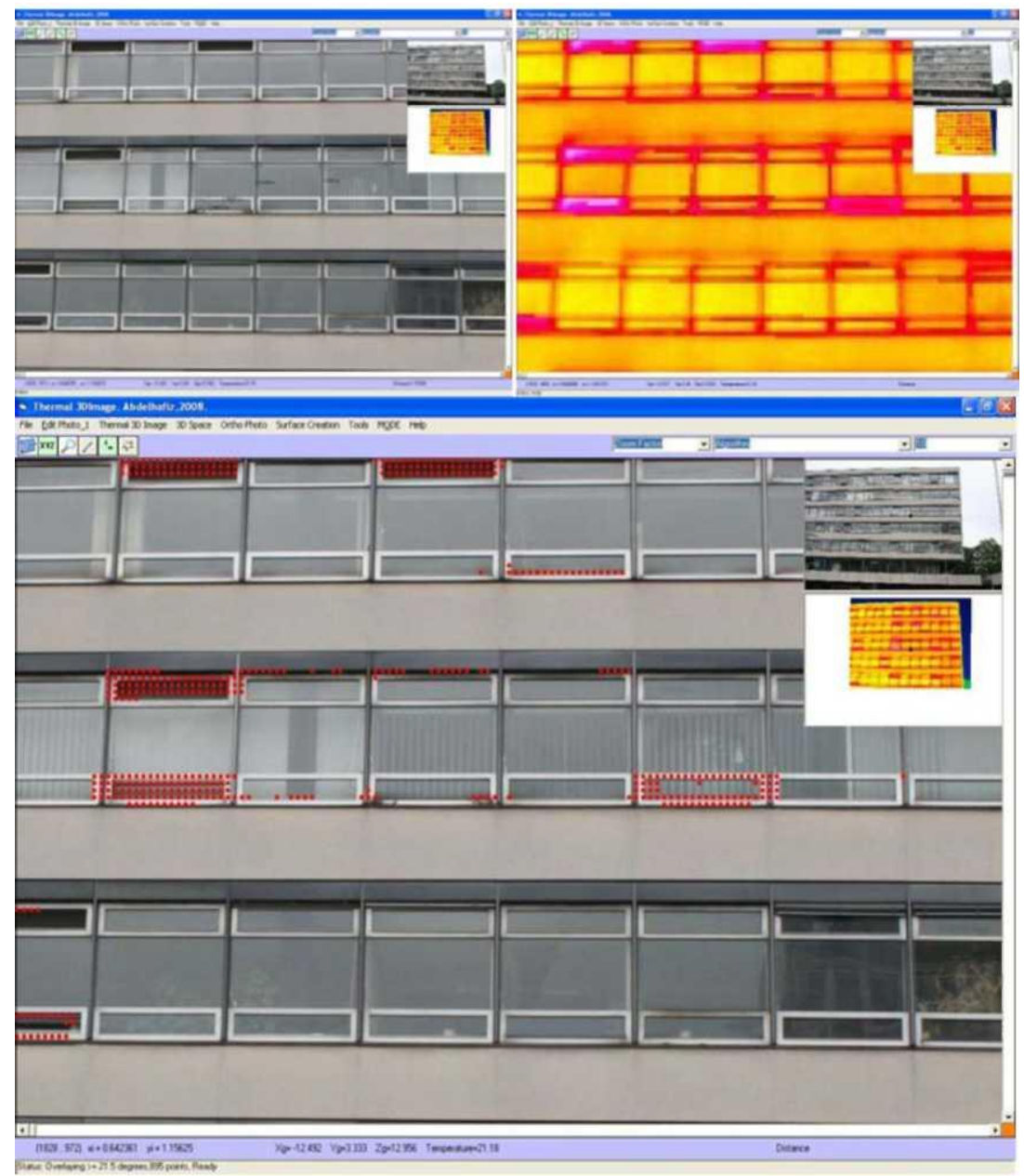

\section{Fig. 10: Thermal3DImage for the main building of the technical university, Braunschweig.}

\section{Conclusions}

In this work, the laser scanner point cloud/mesh, visual images and thermal images are successfully registered and fused. The resulted Thermal3DImage will provide the user with a digital archive file for the concerning building containing all the required information (Temperature / 3D Coordinates / visual views). The following benefits are then obtained in buildings maintenance field:

- Detecting air leakage (heat/cold) coming from buildings openings (doors and windows). Afterwards, the amount of (heat/cold) air can be computed to be checked for the accepted limits.

- Early detection of any expected water seepage which might occur inside buildings walls. Then the exact location of the defected part on the wall can be determined. And consequently the area influenced from the water seepage can be computed. 


\section{References}

[1] Abdelhafiz, A., 2009. Integrating Digital Photogrammetry and Terrestrial Laser Scanning. PhD Thesis, Institute for Geodesy and Photogrammetry, Technical University Braunschweig, Germany. Online on Deutsche Geodaetische Kommission (DGK), Muenchen.

[2] El-Hakim, S. F., Beraldin, J.-A., Picard, M., and Vettore, A., 2003. Effective 3D Modeling of Heritage Sites, Proceedings of 4th International Conference 3D Imaging and Modeling (3DIM'03), 6-10 October, Banff, Canada: 302-309.

[3] Abdelhafiz, A. and Niemeier, W., 2007. Automatic Texturing for Laser Scanner Meshes. Proceedings of 8th Conf. Optical 3D Measurement Techniques, 9-12 July, Zurich, Switherland, CD ROM.

[4] Caprioli, M. and Scognamiglio, A., 2003. Photogrammetry and Laser Scanning in Surveying and 3D Modeling of Architectural Heritage. FIG Working, 13-17 April, Paris, France.

[5] Kern, F., 2003. AutomatisierteModellierung von Bauwerksgeometrien aus 3DLaserscanner-Daten. PhD Thesis, TU Braunschweig, Germany.

[6] Alshawabake, Y., 2006. Integration of Laser Scanning and Photogrammetry for Heritage Documentation. PhD Thesis, Institute of photogrammetry, Stuttgart, Germany.

[7] Remondino, F., Guarnieri, A. and Vettore, A., 2005. 3D Modelling of Close-Range Objects: Photogrammetry or Laser Scanning. Proceedings of SPIE-IS\&T Electronic Imaging: Videometrics VIII, 16-20 January, San Jose, CA: 216-225.

[8] Boehler, W. and Marbs, A., 2004. 3D scanning and photogrammetry for heritage recording: a comparison. Proceedings of the 12th International Conference on Geoinformatics, 7-9 June, Gaevle, Sweden, 291-298.

[9] Kadobayashi, R., Kochi, N., Otani, H. and Furukawa, R., 2004. Comparison and Evaluation of Laser Scanning and Photogrammetry and Their Combined Use for Digital Recording of Cultural Heritage. International Archives of the Photogrammetry, Remote Sensing and Spatial Information Sciences, 35(5): 401- 406.

[10] Maldague, X., 2000. Applications of Infrared Thermography in Non-Destructive Evaluation. In: Trends in Optical Non-destructive Testing, (ed. Pramod Rastogi), Amsterdam, Elsevier.

[11] Gianinetto, M., Roncoroni, F. and Scaioni, M., 2005. Calibration of Close-Range Thermal Imagery for Integration into 3R VR Models. Workshop Italy-Canada '3D Digital Imaging and Modeling: Applications of Heritage, Industry, Medicine and Land', 17-18 May, Padova, Italy. 